\title{
Syndrome de Boerhaave
}

\author{
Fatt Yang Chew MD, Su-Tso Yang MD PhD
}

Citation : CMAJ 2021 September 27;193:E1499. doi : 10.1503/cmaj.202893-f

Voir la version anglaise de l'article ici : www.cmaj.ca/lookup/doi/10.1503/cmaj.202893

$\mathbf{U}$ n homme de 46 ans s'est présenté au service des urgences après 2 épisodes importants de vomissements non sanguinolents et de douleurs abdominales au cours des 3 heures précédentes. II n'avait pas d'antécédents de reflux gastro-œsophagien ni d'autres problèmes médicaux pertinents. Il buvait depuis 20 ans le contenu de 10 à 15 cannettes de bière par semaine. Lors de l'auscultation, son abdomen était rigide et sensible dans le quadrant supérieur gauche. Les résultats d'analyses ont démontré un taux élevé de leucocytes de 13,8 (plage normale $4,5-11,5) \times 10^{9} / \mathrm{L}$ avec $77,8 \%$ de neutrophiles et une concentration de protéine $C$ réactive de haute sensibilité $<0,02$ (plage normale $<0,80) \mathrm{mg} / \mathrm{dL}$. Une radiographie thoracique a révélé un pneumomédiastin (figure $1 \mathrm{~A}$ ), ce qu'une tomodensitométrie thoracique subséquente a aussi démontré ainsi qu'un hydropneumothorax gauche (figure 1B). Nous avons diagnostiqué un Syndrome de Boerhaave, c'est-à-dire une perforation de l'œsophage.

Le patient a subi une réparation œsophagienne et une décortication de la plèvre, accompagnées d'une gastrostomie de drainage et d'une jéjunostomie d'alimentation. Au cours de l'intervention chirurgicale, nous avons découvert une lacération de l'œsophage thoracique inférieur longue de $1,5 \mathrm{~cm}$ (annexe 1, accessible en anglais au www.cmaj.ca/lookup/doi/10.1503/ cmaj.202893/tab-related-content). Seize jours après son opération, nous avons interrompu l'alimentation par jéjunostomie lorsque l'évaluation par œsophagographie a démontré l'absence de fuite d'agent de contraste au site de la réparation. Deux mois plus tard, le patient était complètement rétabli.
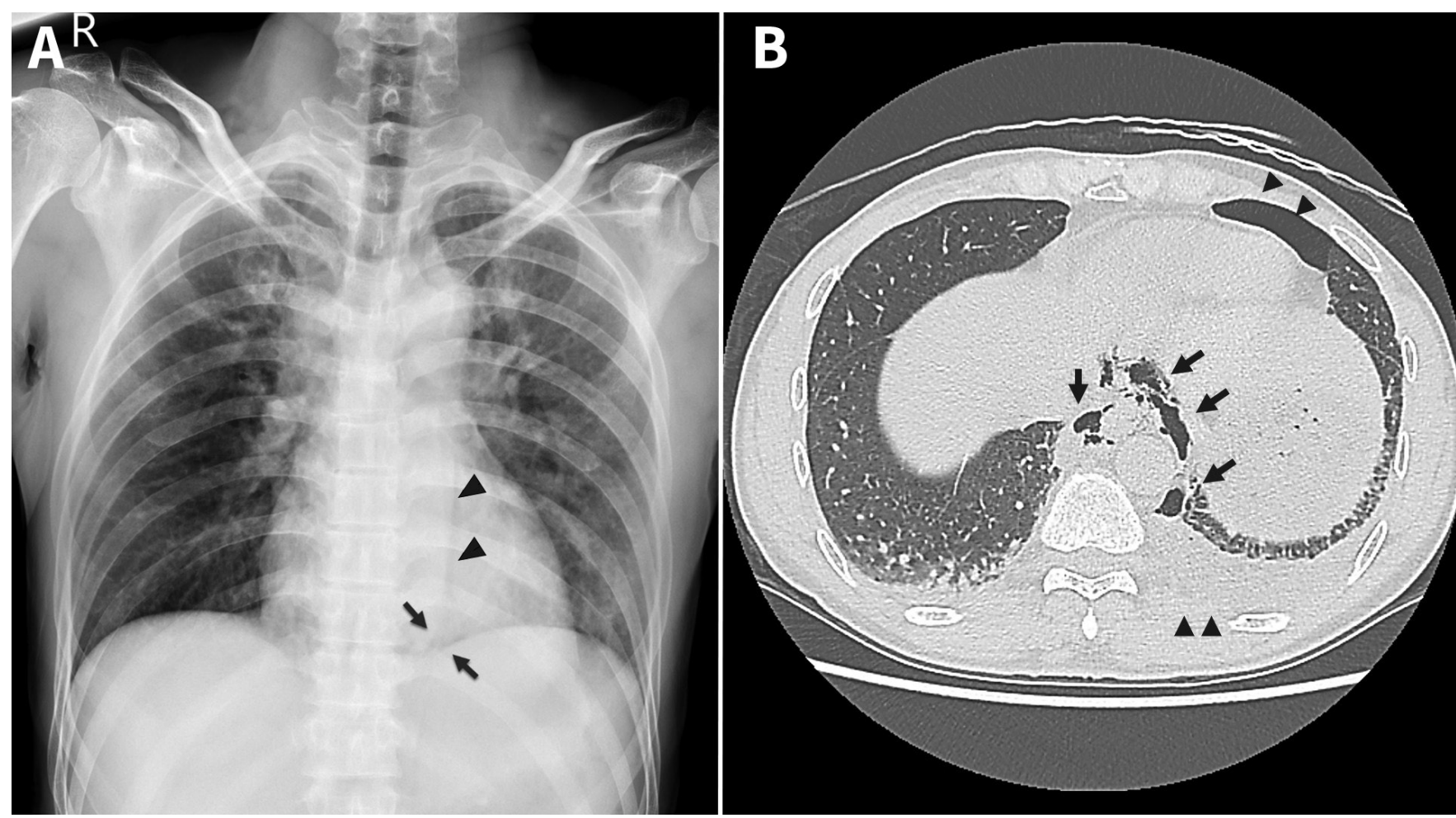

Figure 1 : Syndrome de Boerhaave chez un homme de 46 ans. A) Radiographie thoracique montrant un « $V$ » de Naclério, qui correspond à la présence d'air soulignant le contour médiastinal gauche inférieur latéral (flèches), avec des stries d'air périaortiques latérales de l'aorte thoracique descendante et au-dessus du diaphragme gauche (pointes des flèches). La présence d'un « V » de Naclério confirme un pneumomédiastin. B) Tomodensitométrie thoracique révélant un pneumomédiastin (flèches) et un hydropneumothorax gauche (pointes de flèches) évoquant une rupture œsophagienne. 
Les données épidémiologiques en matière de perforation œsophagienne sont peu nombreuses. Une étude islandaise réalisée à l'échelle du pays a observé une incidence annuelle de 3,1 cas par million de personnes ${ }^{1}$. Une anamnèse et un examen minutieux du patient peuvent aider à déterminer les causes et le site de la perforation. Une revue systématique a révélé que les causes de perforation œsophagienne les plus fréquentes sont iatrogéniques (46,5\%), spontanées (38\%) et le résultat de la présence d'un corps étranger $(6 \%)^{2}$. Plus de $70 \%$ des perforations sont thoraciques, comme dans le cas de notre patient, et les symptômes habituels comprennent des douleurs thoraciques épigastriques et rétrosternales, un souffle court ainsi qu'un emphysème sous-cutané après de forts vomissements ${ }^{2-4}$.

Le Syndrome de Boerhaave est une perforation transmurale de l'œsophage, différente de la déchirure de Mallory-Weiss, laquelle est une lacération non pénétrante des muqueuses de l'œsophage distal, près de la jonction gastro-œsophagienne ${ }^{5}$. On observe plus souvent ces lésions chez les hommes qui consomment des quantités excessives de boissons alcooliques et elles sont associées à un barotraumatisme de l'œsophage; par contre, l'hématémèse s'observe fréquemment dans le syndrome de Mallory-Weiss, mais pas dans celui de Boerhaave ${ }^{5}$. Le traitement dépend de la gravité de la perforation et des études de cas ont décrit des rétablissements après des interventions endoscopiques et chirurgicales conservatrices ${ }^{3}$. Le risque de décès est d'environ $13 \%$.

\section{Références}

1. Vidarsdottir H, Blondal S, Alfredsson $\mathrm{H}$, et al. Oesophageal perforations in Iceland: a whole population study on incidence, aetiology and surgical outcome. Thorac Cardiovasc Surg 2010;58:476-80.

2. Sdralis EIK, Petousis S, Rashid F, et al. Epidemiology, diagnosis, and management of esophageal perforations: systematic review. Dis Esophagus 2017;30:1-6.

3. de Schipper JP, Pull ter Gunne AF, Oostvogel HJM, et al. Spontaneous rupture of the oesophagus: Boerhaave's syndrome in 2008. Literature review and treatment algorithm. Dig Surg 2009;26:1-6.

4. Sancheti MS, Fernandez FG. Surgical management of esophageal perforation. Oper Tech Thorac Cardiovasc Surg 2016;20:234-50.

5. Cuccì M, Caputo F, Fraternali Orcioni G, et al. Transition of a Mallory-Weiss syn drome to a Boerhaave syndrome confirmed by anamnestic, necroscopic, and autopsy data: a case report. Medicine (Baltimore) 2018;97:e13191.

Intérêts concurrents : Aucun déclaré.

Cet article a été révisé par des pairs.

Les auteurs ont obtenu le consentement du patient.

Affiliations : Département d'imagerie médicale (Chew, Yang), Hôpital de l'université de médecine de Chine et École de médecine chinoise (Yang), Collège de médecine chinoise, Taichung, Taiwan.

Propriété intellectuelle du contenu : Il s'agit d'un article en libre accès distribué conformément aux modalités de la licence Creative Commons Attribution (CC BY-NC-ND 4.0), qui permet l'utilisation, la diffusion et la reproduction de tout médium à la condition que la publication originale soit adéquatement citée, que l'utilisation se fasse à des fins non commerciales (c.-à-d., recherche ou éducation) et qu'aucune modification ni adaptation n'y soit apportée. Voir : https://creativecommons.org/licenses/by-nc-nd/4.0/deed.fr.

Correspondance : Fatt Yang Chew, c_fyang@hotmail.com 